\title{
A pilot study of a low glycemic load diet in patients with stage I-III colorectal cancer
}

\author{
Michelle Treasure ${ }^{1}$, Alicia Thomas ${ }^{2}$, Stephen Ganocy ${ }^{3}$, Augustine Hong ${ }^{4}$, Smitha S. Krishnamurthi ${ }^{1}$, \\ David L. Bajor ${ }^{5}$, Nathan A. Berger ${ }^{6}$, Neal J. Meropol ${ }^{7,8}$
}

${ }^{1}$ Department of Medicine, Division of Hematology and Oncology, Cleveland Clinic Foundation, Case Comprehensive Cancer Center, Cleveland Clinic Learner College of Medicine, Cleveland, OH, USA; ${ }^{2}$ Case Western Reserve University School of Medicine, University Hospitals Cleveland Medical Center, Cleveland, OH, USA; ${ }^{3}$ Department of Psychiatry, University Hospitals Cleveland Medical Center, Case Western Reserve University, Cleveland, OH, USA; ${ }^{4}$ Department of Medicine, University Hospitals Cleveland Medical Center, Case Western Reserve University, Cleveland, OH, USA; ${ }^{5}$ Department of Medicine, Division of Hematology and Oncology, University Hospitals Cleveland Medical Center, Case Comprehensive Cancer Center, Case Western Reserve University School of Medicine, Cleveland, OH, USA; ${ }^{6}$ Department of Medicine, Division of Hematology and Oncology, University Hospitals Cleveland Medical Center, Case Comprehensive Cancer Center, Case Western Reserve University School of Medicine, Cleveland, OH, USA; ${ }^{7}$ Case Comprehensive Cancer Center, Case Western Reserve University School of Medicine, Cleveland, OH, USA; ${ }^{8}$ Flatiron Health, Inc., New York, NY, USA

Contributions: (I) Conception and design: M Treasure, NJ Meropol, NA Berger, A Thomas; (II) Administrative support: None; (III) Provision of study materials or patients: M Treasure, A Thomas, NJ Meropol, SS Krishnamurthi, DL Bajor; (IV) Collection and assembly of data: M Treasure, A Thomas, A Hong; (V) Data analysis and interpretation: S Ganocy, M Treasure, NJ Meropol, NA Berger; (VI) Manuscript writing: All authors; (VII) Final approval of manuscript: All authors.

Correspondence to: Michelle Treasure, MD. Cleveland Clinic Foundation, Department of Medicine, Division of Hematology and Oncology, Moll Cancer Center at Fairview Hospital, 18200 Lorain Road, Cleveland, OH 44111, USA. Email: treasum@ccf.org.

Background: Consumption of a diet with high glycemic indices has been associated with inferior cancerspecific outcomes in patients with early-stage colorectal cancer, but there is limited prospective evidence that alterations in dietary habits improves cancer outcomes. This study aimed to determine the feasibility and acceptability of following a low glycemic load (GL) diet in patients with stage I-III colorectal cancer.

Methods: Patients with stage I-III colorectal cancer, who completed definitive therapy, and consumed an average daily GL > 150 participated in a 12-week tailored face-to-face dietary intervention with a target GL. This study followed a 2 -stage design, with 4 planned cohorts, each with an assigned GL target and dietary intervention intensity. The primary endpoint of feasibility was determined by participant compliance, defined as an individual following the assigned GL $\geq 75 \%$ of the time. Compliance was determined using 24-hour telephone recalls. A cohort was deemed feasible if at least $67 \%$ of participants were compliant. Secondary endpoints included acceptability of the diet, nutritional support resources necessary to follow the diet, and evaluation of the effect of the diet on physical measures and correlative laboratories.

Results: Only cohort 1 was required as the primary endpoint of feasibility was met (stringent GL target, low intensity dietary support). The majority of participants experienced a decrease in body mass index (BMI) and waist circumference, $29 \%$ experiencing meaningful weight loss $(\geq 5 \%)$. The dietitian spent an average of 6.97 hours (SD 2.18) face-to-face time and 1.58 hours (SD 0.68) by phone with each participant. Significant decreases were seen in total cholesterol, very-low-density lipoprotein (VLDL) and triglycerides (all $\mathrm{P}<0.05$ ). All participants liked the foods and were satisfied with the diet. All participants felt the in-person meetings were helpful, and $62 \%$ did not feel a virtual meeting (e.g., Skype, etc.) could replace in-person meetings.

Conclusions: Patients with stage I-III colorectal cancer can follow a low GL diet with a 12-week inperson dietary intervention. Significant changes in physical and laboratory measures suggest relevant biologic effects of the dietary intervention. This study establishes feasibility, and warrants a larger scale prospective intervention trial to evaluate the impact of a low GL diet on cancer outcomes. 
Keywords: Glycemic load (GL); colorectal cancer; early stage

Submitted Aug 21, 2020. Accepted for publication Mar 28, 2021.

doi: 10.21037/jgo-20-330

View this article at: http://dx.doi.org/10.21037/jgo-20-330

\section{Introduction}

Colorectal cancer is the second leading cause of cancer death in men and the third leading cause of death in women the United States each year (1). Despite advances in adjuvant therapy, observed 5 -year overall survival in patients with stage III disease range from $12.9 \%$ to $73.7 \%$ (2) depending on the tumor and nodal pathologic features, with average survival of $71.8 \%(2-5)$. In those with 4 or more lymph nodes, greater than $50 \%$ will recur or die within 5 years of diagnosis (6). While advances in molecular profiling of tumors have provided an opportunity to tailor adjuvant therapy, attempts to add targeted therapies to an adjuvant chemotherapy backbone of fluoropyrimidine plus oxaliplatin have failed to improve survival (7-9). This raises the question of what factors, in addition to inherent tumor characteristics, influence recurrence risk; largely unaddressed are the influences of behavioral factors on micrometastases.

Epidemiologic research clearly associates diet and other lifestyle habits with the risk of developing colon cancer. Beyond increasing the risk for malignancy, preclinical and clinical data suggest these modifiable lifestyle factors that affect energy balance, in particular diet, may impact outcomes in those definitively treated for colon cancer $(6,10-13)$ through effects on the extracellular environment influencing tumor cell survival. Thus dietary interventions offer potential alternative therapeutic strategies.

An observational study has shown that consumption of a Western diet, known to consist of foods with high glycemic indices, negatively impacts both progression free and overall survival in patients with stage III colon cancer who are treated with standard adjuvant therapy, most notable in those with body mass index (BMI) $>25$ (HR 2.26) $(6,10)$. The glycemic index (GI) is a unit of measurement which stratifies carbohydrates based on postprandial blood glucose response. The value is dependent on the nature of the carbohydrate compared to a reference food, either white bread or glucose. A related measurement is the glycemic load (GL) which accounts for the quantity of food consumed.

Postprandial blood glucose responses are felt to influence carcinogenesis through several mechanisms including hyperinsulinemia and disruptions in the insulin like growth factor (IGF) axis, influencing tumor cell growth, differentiation, and apoptosis (10,14-19). Additionally, alterations in glucose metabolism in cancer cells (Warburg effect) is felt to provide selective growth advantage (20). Dietary habits also affect lipid metabolism which influences the development and outcomes of colorectal malignancy $(21,22)$. The increase in adverse cancer related outcomes observed in those with a BMI $>25$, is felt to be related to the pleiotropic effects of obesity on pro-oncogenic mediators of cellular metabolism, involving the aforementioned mechanisms, as well as alterations in adipokine secretion and subclinical inflammation (14,23-27). The site of adiposity storage may also influence colorectal cancer outcomes, as increased waist circumference has been associated with increased recurrence risk $(27,28)$.

We hypothesize that reducing dietary GL in the adjuvant setting will improve survival in patients with locoregional colon or rectal cancer. As an initial step in testing this hypothesis prospectively, it is necessary to demonstrate the feasibility of following a low GL diet in this patient population and determine the necessary resources to achieve compliance. Our intervention consisted of in-person contact (educational and supportive resources) with a dietitian, given evidence that dietitian-led and tailored interventions increase dietary compliance (29-31). We also evaluated the dietitian service resources necessary to facilitate adherence to and acceptability of the diet. In correlative analysis we evaluated the effect of lowering the dietary GL on BMI, lipid metabolism and other pro-oncogenic intermediaries of cellular metabolism. We present the following article in accordance with the TREND reporting checklist (available at: http://dx.doi.org/10.21037/jgo-20-330).

\section{Methods}

The study was approved by the Institutional Review Board at Seidman Cancer Center, University Hospitals Cleveland Medical Center, and utilized the Reliant Review at Metrohealth Medical Center (University Hospitals 01-14- 
07C, MetroHealth 15-00732). The study was conducted in accordance with the Declaration of Helsinki (as revised in 2013).

\section{Subjects}

Eligible patients were 18 years or older, English speaking, with a history of stage I-III colon or rectal cancer who had undergone definitive therapy (surgery with or without chemotherapy and/or radiation therapy), and regularly consumed a diet with a daily GL (using white bread reference) $>150$, as estimated through a 3 -day self-reported food record. They also needed to be readily available for a 3-month period and agreeable to participate in regular dietary adherence assessments.

Participants were identified through medical and surgical oncology clinic referrals as well as through the tumor registries at University Hospitals Cleveland Medical Center and MetroHealth Medical Center in Cleveland, Ohio. Patients were approached at the time of their follow up visits or contacted by phone. Those identified through the tumor registry were contacted by phone and if interested were mailed a consent form and a 3-day dietary food record. They were then contacted to review the consent form and if interested, consented by phone and were contacted by a member of the bionutrition team, who provided detailed instructions on how to complete a 3-day dietary intake log and return it for screening and evaluation. If eligible, an appointment with the dietitian was made either alone or in a small group, and in-person written informed consent was obtained at that time for each participant.

\section{Instruments and intervention}

This was a "dose escalation" study with four planned cohorts each with an assigned GL target using white bread as a reference (Stringent $\leq 102 \mathrm{GL}$ or Lenient between 126-150 GL) and dietary intervention intensity (Low intensity or High intensity). The first cohort was our ideal intervention with the lowest GL target $(\leq 102)$ and the minimal nutritional service support. Subsequent cohorts either relaxed the GL target or increased the dietitian service support. Each cohort was intended to follow a 2 -stage study design with early termination of a cohort if the primary endpoint of feasibility was not met, and plan to close once the feasibility endpoint was met (schema included in supplemental material). The sample size in the first stage was $n=12$ and if $\geq 8$ participants were compliant with the diet, 18 patients were enrolled in that cohort. Feasibility was determined by participant compliance which was defined as individual following the assigned target GL $\geq 75 \%$ of the time between weeks 4 and 12 . Compliance was determined through use of 24-hour telephone recalls every 2 weeks at random and calculating the GL. The cohort was deemed feasible if $67 \%$ of participants were compliant. Secondary endpoints included acceptability of the diet assessed though use of a Food Acceptability Questionnaire, and evaluation of the effect of the diet on physical measures including BMI and waist circumference, lipid metabolism and pro-oncogenic intermediaries of cellular metabolism, which were assessed monthly.

Only cohort 1 was required as our primary endpoint of feasibility was met. In this cohort, participants met in person with the dietitian for the initial visit and were given verbal and written education materials, including food tables with glycemic indices, recipes, portion size information, meal plans, food preparation and grocery shopping instruction. Individual instruction was tailored to each participant's baseline dietary preferences (e.g., vegan, allergies, etc.) and effort was made to maintain a heart healthy diet. Individualized food preferences were able to be maintained throughout the study. As the GL is impacted by each food's GI along with the quantity consumed, participants choosing higher GI foods were counseled on portion sizes for those items. Participants then had face-to-face contact with the dietitian every 2 weeks, and phone contact on alternating weeks for a total of 12 weeks. A calendar of study events is shown in Table 1.

Dietary food logs were used to determine participant eligibility. This valid dietary assessment tool allowed for real time calculations of each participant's average daily GL. Because individual intake can vary day-to-day, dietary intake and GL was averaged over a 3-day period. Participants completed a self-reported dietary log that consisted of 1 weekend day, 2-week days, and were non-consecutive days. Three 24-hour dietary records are routinely used to assess intake and considered an optimal amount of time for estimation of macronutrients (32). In order to minimize common errors with self-reported dietary recalls, such as under-reporting, detailed oral and written instructions were reviewed with participants by a dietitian, including instruction on how to choose the days. All food and beverage entries were reviewed with the participant for clarity and portion sizes. Participants were eligible they consumed a diet with an average daily GL $\geq 150$.

The first group meeting consisted of a 90-minute 
Table 1 Study calendar

\begin{tabular}{|c|c|c|c|c|c|c|c|c|c|c|c|c|c|}
\hline Variables & wo & W1 & W2 & W3 & W4 & W5 & W6 & W7 & W8 & W9 & W10 & W11 & W12 \\
\hline Phone contact with nutritionist & & $x$ & & $x$ & & $x$ & & $x$ & & $x$ & & $x$ & \\
\hline Correlative laboratories & $x$ & & & & $x$ & & & & $x$ & & & & $x$ \\
\hline Height & $x$ & & & & & & & & & & & & \\
\hline Waist circumference & $x$ & & & & & & & & & & & & $x$ \\
\hline 24 hour phone dietary recall & & & & & $x$ & & $x$ & & $x$ & & $x$ & & $x$ \\
\hline FAQ & & & & & & & $x$ & & & & & & $x$ \\
\hline Program evaluation survey & & & & & & & & & & & & & $x$ \\
\hline
\end{tabular}

education session with the dietitian, which included details about diet and compliance, recipes specific to low GL, handouts on portion sizes for more accurate documentation of intake, food tables of common foods with GL values, food records to log and document intake and contact information of the bionutrition team. All education items were orally reviewed and provided in written format in an organized folder for each participant. Groups varied in size from groups of 1-4 people. Participants did not get to choose their group size, and group participants were random based on the timing of their enrollment. It was attempted to get at least 3 participants in a group.

Subsequent follow-up group education sessions occurred at weeks $2,4,6,8,10$, and 12, and averaged 60 minutes in duration. Group sessions consisted of review of dietary intake logs, how to maintain a healthy diet with respect to low fat, low sodium, and low added sugar, low GLnutrient dense foods, helpful hints while eating out, recipe modifications, general nutrition, diet and disease prevention, importance of support partners, and lastly, individualized answers to participant's questions.

Telephone follow-up calls, conducted by a dietitian, were attempted during weeks $1,3,5,7,9$, and 11. These consisted of a 24-hour dietary recall, and reminder of attendance at next group meeting.

The twenty-four-hour dietary recall was used to assess patient compliance, and was based upon use of self-reported detailed dietary logs, which the dietitian reviewed inperson and by phone. Dietary intake data were collected and analyzed using Nutrition Data System for Research (NDSR) software version 2016, developed by the Nutrition Coordinating Center (NCC), University of Minnesota, Minneapolis, MN. NDSR is a comprehensive, gold-standard research software program and database and includes GL calculations (33-35). A standardized multiple-pass approach for collecting dietary recalls is utilized in NDSR software, allowing ample opportunities for participants to remember intake from the previous day. Implementing 24-h dietary recalls for compliance in dietary intervention studies is frequently utilized in epidemiologic studies (36).

The Food Acceptability Questionnaire was used to assess the acceptability of the diet at weeks 6 and 12. It is a 12 -item questionnaire, which uses a 7-point Likert scale to rate ease of preparation, palatability, satisfaction, perceived benefits or adverse effect of a particular diet. This tool has strong face validity but has not been extensively tested (37-40). It was a self-administered, paper survey.

A 14-item program evaluation, self-administered paper survey, was offered at the end of the 12-week intervention to assess participant satisfaction with various aspects of the intervention and to elicit suggestions on how to improve the intervention. This instrument included 3 yes/no questions with the opportunity to explain choice, 3 multiple choice questions on preference of frequency and duration of visits, 3 Likert questions on helpfulness of in-person visits, phone calls and likelihood to continue to follow the diet. It included one 10 -point scale question on confidence to continue to follow the diet. There were also 4 qualitative items where participants could offer what they liked best, least, resources they would have liked, and open-ended suggestions.

\section{Statistical analysis}

Descriptive analyses were used to describe the study sample, changes in BMI and waist circumference, food acceptability score and also program evaluation results. 


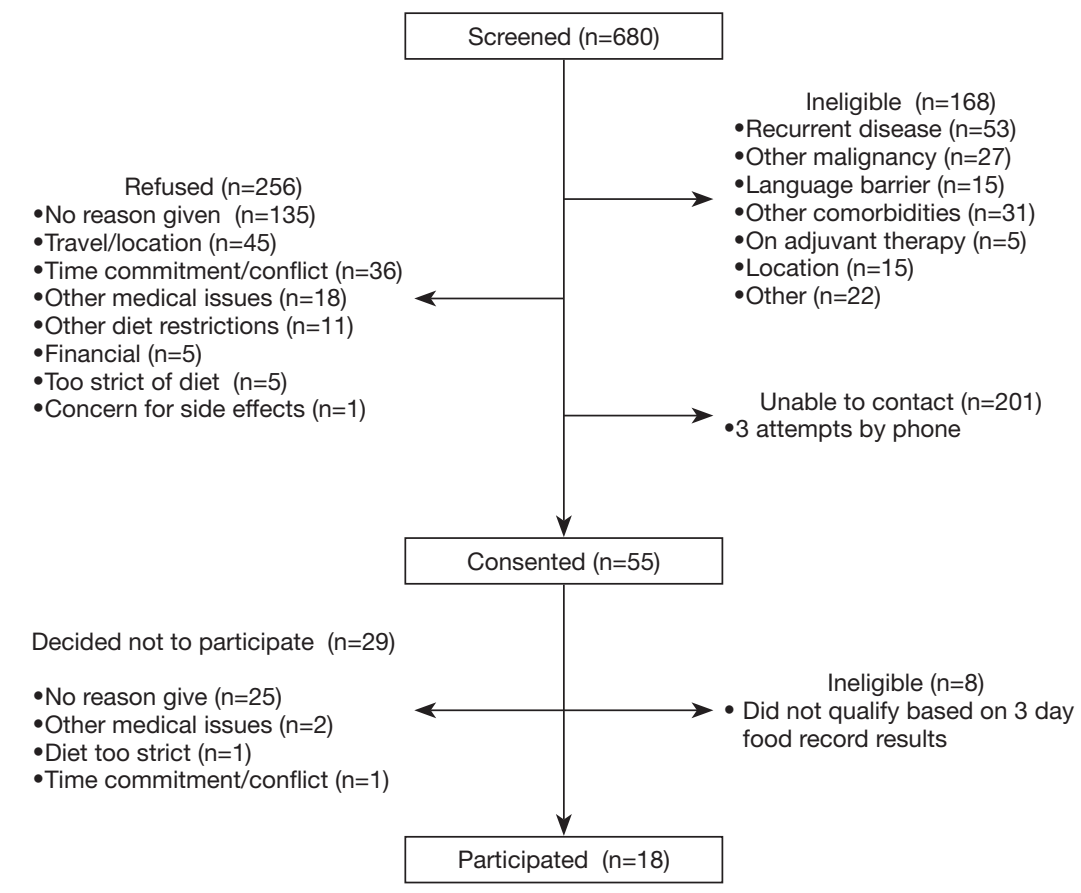

Figure 1 Patient enrollment diagram.

Exploratory correlative analyses evaluated the change in serum levels of glycosylated hemoglobin, markers of lipid metabolism, including total cholesterol, triglycerides, low density lipoprotein (LDL) high density lipoprotein (HDL) and leptin, and serum levels of proteins and metabolites affected by carbohydrate metabolism including fasting glucose, c-peptide, insulin-like growth factor peptides levels [including IGFs (IGF I and IGF II) and their binding proteins (IGFBP1-IGFBP6)]. The difference from baseline to week 12 was assessed using a paired $t$-test.

\section{Results}

Eighteen patients enrolled in this study. One patient withdrew from the study and was not included in the analysis and one patient did not complete the 12-week intervention and was included in the analysis. Figure 1 provides information on recruitment. Patient demographic and clinical characteristics are summarized in Table 2. Sex, race and ethnicity were self-reported.

The primary endpoint of feasibility was met in that $67 \%(n=12)$ of participants were compliant with the diet over the 12-week intervention period. Compliance was not associated with sex, race, income, or tumor stage.

Weight change data were available on 14 patients. Of those included in the analysis, $79 \%(\mathrm{n}=11)$ of participants lost weight, $14 \%(n=2)$ gained weight, and $7 \%(n=1)$ maintained weight. Twenty eight percent of patients were considered to have meaningful weight loss, defined as $\geq 5 \%$. Overall there was a statistically significant decrease in BMI, with a mean decrease of 1.2 (SEM 0.3) $\mathrm{P} \leq 0.01$. The average weight decrease in the compliant group was $4.13 \mathrm{~kg}$ (SD 2.49) and $1.98 \mathrm{~kg}$ (SD 5.53) in the non-compliant group $(\mathrm{P}=0.32)$. While this difference was not statistically significant, the trend was in the anticipated direction.

Among 13 patients with serial waist circumference measurements, five $(62 \%)$ had a decrease in waist circumference, two (15\%) had no change and three (23\%) had an increase in waist circumference. The mean change in waist circumference in the compliant group was $-3.92 \mathrm{~cm}$ (SD 5.25) and was $+0.69 \mathrm{~cm}$ (SD 7.50) in the non-compliant group $(\mathrm{P}=0.224)$.

\section{Nutrition resources}

The dietitian spent an average of 6.97 hours (SD 2.18) face-to-face and 1.58 hours (SD 0.68) by phone with each participant over 12 weeks. The dietitian recorded the time spent at each in-person and phone session and this was averaged for each participant. 
Table 2 Patient characteristics

\begin{tabular}{|c|c|}
\hline Characteristic & N (\%) \\
\hline Age (year): median [range] & $59[37.5,82.5]$ \\
\hline \multicolumn{2}{|l|}{ Sex } \\
\hline Female & $8(47.1)$ \\
\hline Male & $9(52.9)$ \\
\hline \multicolumn{2}{|l|}{ Race } \\
\hline Black & $5(29.4)$ \\
\hline White & $12(70.6)$ \\
\hline \multicolumn{2}{|l|}{ Ethnicity } \\
\hline Non-Hispanic & $16(94.1)$ \\
\hline Not stated & $1(5.9)$ \\
\hline \multicolumn{2}{|l|}{ Income per year } \\
\hline$<\$ 20 K$ & $3(17.7)$ \\
\hline$\$ 21 \mathrm{~K}-\$ 50 \mathrm{~K}$ & $5(29.4)$ \\
\hline$>\$ 50 \mathrm{~K}$ & $9(52.9)$ \\
\hline \multicolumn{2}{|l|}{ Education } \\
\hline High school & $1(5.9)$ \\
\hline Some college & $5(29.4)$ \\
\hline Associate degree & $3(17.7)$ \\
\hline Bachelor degree & $5(29.4)$ \\
\hline Past-graduate & $3(17.7)$ \\
\hline \multicolumn{2}{|l|}{ Adjuvant therapy } \\
\hline Yes & $8(47.1)$ \\
\hline No & 9 (52.9) \\
\hline \multicolumn{2}{|l|}{ Stage } \\
\hline I & $5(29.4)$ \\
\hline$\| A$ & $6(35.3)$ \\
\hline IIIA & $4(23.5)$ \\
\hline IIIB & $2(11.9)$ \\
\hline \multicolumn{2}{|l|}{ Diabetes } \\
\hline No & $13(76.5)$ \\
\hline Yes & $4(23.5)$ \\
\hline
\end{tabular}

\section{Acceptability of the diet}

There were no significant changes in response to the food acceptability measure between 6 and 12 weeks. At 12 weeks when asked "Overall how satisfied or dissatisfied are you with this diet?" all patients were satisfied to some extent and $43 \%(n=7)$ were extremely satisfied. For the question "How well do you like these foods?" all participants answered moderately or better and $75 \%(\mathrm{n}=12)$ liked the foods very much or extremely. When asked "How easy or difficult has it been for you to prepare these foods?" $93 \%(n=15)$ answered neutral to extremely easy; the median and mode response was 6 (extremely easy). When asked "How easy or difficult has it been for you to purchase these foods?" $75 \%$ $(\mathrm{n}=12)$ answered neutral to extremely easy, with 5 (fairly easy) as the most common response. Participants were asked to describe symptoms experienced over prior 2 weeks, $63 \%$ $(\mathrm{n}=10)$ reported increased energy, 50\% $(\mathrm{n}=8)$ felt improved digestion, and $19 \%(n=3)$ better sleep than usual.

\section{Correlative lab studies}

Over the course of the dietary intervention, there were significant changes in several lipid metabolites, including a $7.2 \%$ decrease in the mean total cholesterol, a $26.8 \%$ decrease in very-low-density lipoprotein (VLDL), and a $26.6 \%$ decrease in triglycerides between baseline and week 12 (Table 3). There were no other statistically significant differences in biomarkers over the 12 -week time period, though point estimates in several biomarkers were in the anticipated direction of change, including IGF2, IGFBP1 and 2, leptin, and LDL. When evaluating changes in biomarkers between the compliant and non-compliant groups there was significant change in IGFBP3 (absolute difference in IGFBP 3 was $293 \mathrm{ng} / \mathrm{mL}, \mathrm{t}=2.44, \mathrm{P}=0.03$ ). In the group that was compliant with the diet the mean increase was $71.1 \mathrm{ng} / \mathrm{mL}$ and the group that was not compliant with the diet the mean decrease was $221.8 \mathrm{ng} / \mathrm{mL}$. There were sizable differences in mean IGFBP2, and Insulin levels, though they did not meet statistical significance $(t=1.15$, $\mathrm{P}=0.14 ; \mathrm{t}=-1.51, \mathrm{P}=0.15)$.

\section{Program evaluation}

When asked if they liked the group size, all participants responded "yes" and felt the frequency of in person visits every 2 weeks was "just right." Ninety-two percent thought the duration of meetings were "just right" and one person felt they were "too short." Eighty-six percent of participants felt the follow up phone calls were helpful to some degree "a little helpful" to "extremely helpful." All patients felt the in-person meetings were "moderately" to "extremely 
Table 3 Changes in potential biomarker and physical measurements between baseline and week 12

\begin{tabular}{|c|c|c|c|c|}
\hline Variables & Baseline, mean (SEM) & Week 12, mean (SEM) & Change, mean (SEM) & $\mathrm{t}, \mathrm{P}$ value \\
\hline Total cholesterol (mg/dL) & $179.4(13.2)$ & $166.6(12.1)$ & $-12.8(5.5)$ & $-2.33,0.03$ \\
\hline VLDL (mg/dL) & $24.6(3.4)$ & $18.0(1.2)$ & $-6.6(2.8)$ & $-2.33,0.03$ \\
\hline Triglycerides (mg/dL) & $122.6(17.0)$ & $90.0(6.0)$ & $-32.6(14.2)$ & $-2.29,0.04$ \\
\hline IGF $2(\mathrm{pg} / \mathrm{mL})$ & $402,432[23,657]$ & $392,420[23,742]$ & $-10,012[10,224]$ & $-0.98,0.34$ \\
\hline IGFBP1 (pg/mL) & $14,853[2,186]$ & $17,401[2,474]$ & $2,548[1,849]$ & $1.38,0.18$ \\
\hline IGFBP2 (ng/mL) & 246.1 (31.2) & $271.2(37.7)$ & $25.1(23.7)$ & $1.06,0.30$ \\
\hline IGFBP3 (ng/mL) & $1,913[136]$ & $1,898[148]$ & $-15(62.7)$ & $-0.24,0.81$ \\
\hline Hgb A1c (\%) & $5.9(0.2)$ & $5.8(0.1)$ & $-0.1(0.1)$ & $-1.23,0.24$ \\
\hline Glucose (mg/dL) & $111.8(6.7)$ & $107.2(4.8)$ & $-4.6(3.7)$ & $-1.25,0.23$ \\
\hline Insulin (ulU/mL) & $13.3(2.3)$ & $13.8(2.7)$ & $0.5(2.0)$ & $0.27,0.79$ \\
\hline $\mathrm{HDL}(\mathrm{mg} / \mathrm{dL})$ & $54.6(4.0)$ & $54.2(3.7)$ & $-0.3(1.6)$ & $-0.21,0.84$ \\
\hline HDL ratio & $3.4(0.2)$ & $3.1(0.1)$ & $-0.3(0.2)$ & $-1.55,0.14$ \\
\hline \multicolumn{5}{|l|}{ Physical measurements } \\
\hline BMI $\left(\mathrm{kg} / \mathrm{m}^{2}\right)$ & $31.3(2.0)$ & $29.9(2.1)$ & $-1.2(0.3)$ & $-3.8,<0.01$ \\
\hline
\end{tabular}

VLD, very-low-density lipoprotein; IGF, insulin like growth factor; HDL, high density lipoprotein; BMI, body mass index.

helpful." Seventy-seven percent did not feel an online video could replace the in-person meetings. When asked about using Skype/virtual visits, $62 \%$ of participants did not feel a virtual meeting could replace the in-person meeting while $38 \%$ felt it could. All patients responded that they were very likely or extremely likely to follow the diet after completion of the study, and in regards to how confident they were in their ability to follow the diet using a scale of 1 to 10 , all patients answered between 6-10, median 8 and mode 7 .

\section{Discussion}

The results of this pilot study suggest that a low GL diet, with face-to-face dietitian support, is feasible and acceptable for colorectal cancer survivors. Biomarkers relevant to energy balance also showed directional changes consistent with the dietary intervention.

A major issue with behavioral modification is the sustainability of the prescribed lifestyle habit once the intervention contact has stopped. There are several studies showing rapid attenuation of dietary habits after cessation of the intervention. We used a face-to-face dietary intervention given literature on increased compliance with diet alterations with this method as compared to other modalities such as phone, mail or other written educational material (30). The majority of patients in our study reported they planned to continue with the diet after completion of the study and felt comfortable doing so on their own with the training they received. One of the study limitations was the lack of follow up to assess longer-term dietary habits. However, several patients continued to have contact with the study dietitian and reported continuing the diet with encouragement from their other clinicians to continue their lifestyle changes as improvements were noted in control of their other comorbid conditions including diabetes and hypertension. Several participants reported that other family members were also following the diet plan. One subject routinely sends new low GL recipes to the dietitian.

Of the potential biomarkers evaluated, in the group overall, there was statistically significant decrease in the 
mean total cholesterol, VLDL and triglycerides between baseline and week 12. Dyslipidemia is strongly related to insulin resistance and the aforementioned pathophysiology pathways involving hyperinsulinemia and alterations in the IGF axis $(21,22)$. There are several other proposed mechanisms linking altered lipid metabolism and cancer risk and possibly outcomes, including observed correlations between various lipids and pro-inflammatory cytokines such as TNF alpha and IL-6 (41). However, lipid components may serve as markers of other lifestyle exposures, which may be the actual modifiers of cancer risk and outcome. The clinical significance of alterations in lipid metabolism after the diagnosis of cancer remains uncertain, and lipidomic studies and their associations with colorectal cancer outcomes are an active area of research.

When comparing the compliant versus non-compliant group, there was a significant difference in the change in IGFBP3, with increased level in the compliant group and decreased levels in the non-compliant group. There were sizable point differences in the change in IGFBP2, and Insulin levels, though they did not meet statistical significance. The role of IGFBP3 in carcinogenesis remains unclear, as studies have had conflicting results $(14,15,18,42)$, but increased levels may be beneficial as IGFBP 3 binds greater than $95 \%$ of the circulating IGF, modulating its access to IGF receptors and influencing cell proliferation $(18,42)$. It has also been shown to influence growth and apoptosis through mechanisms independent of IGF (42). Conclusions on the effect of the diet on potential biomarkers were limited by the small sample size and short duration of our study. Another limitation was that we did not collect data on activity level which may have influenced the exploratory correlative laboratory results.

Given the complex and interdependent nature of various energy balance factors it is difficult to determine the impact of altering individual factors. Additionally, the degree to which each factor influences the biology of the tumor, whether their influence is fixed and prognostic or dynamic and potentially altered by lifestyle changes, and whether they influence the response to tumor-directed therapies is uncertain. The observed changes in physical and laboratory measures suggest possible biologic effect of the dietary intervention, but these data must be viewed as exploratory and hypothesis-generating, and a large scale prospective study is necessary to determine if these changes affect outcomes in those already diagnosed with colorectal cancer, and if the benefit differs by baseline phenotypes, i.e., those with increased baseline BMI.
To further inform the design of a future large scale study of the impact of a low GL diet on colorectal cancer recurrence, we elicited participant satisfaction with the diet and the intervention, as well as suggestions on how to improve the intervention. All patients were satisfied with the diet and the majority were "extremely satisfied." This may have in part been secondary to the personalized training each participant received, which was felt to be one of the strengths of the intervention, as well as this being a selfselected population. Seventy-five percent of patients liked the foods they were able to eat very much and the majority felt the meals were easy to prepare and purchase. The flexibility of the diet and fact that participants were able to continue to eat foods they enjoyed in moderation was an important component of the success and acceptability of the diet. The majority of patients also felt the diet positively impacted their energy, and half the patients felt the diet positively impacted their digestion. While most patients felt the face-to-fact interaction was important in their success, nearly 40 percent felt a virtual meeting could have replaced the face-to-face meetings. We note that some patients who provided verbal consent to participate by phone, subsequently withdrew, suggesting that program enhancements might be required to broaden the appeal of a dietary intervention. In this regard, virtual telehealth is an opportunity that warrants further evaluation, given the time and resources necessary for in-person sessions and the recent large scale adoption of various virtual exercise programs as well as telehealth, which has quickly advanced due to efforts to safely evaluate and treat patients during the COVID-19 pandemic $(43,44)$.

This study was an essential first step in demonstrating the feasibility and acceptability of following a low GL diet in the patient population. Additional prospective study is necessary to determine if purposeful dietary changes after the diagnosis of colorectal cancer changes cancer-specific clinical outcomes such as risk of recurrence and overall survival. The diagnosis of cancer, like many other health conditions, is often a transformative event during which people are more likely to implement recommendations to change life style habits. This study suggests that colorectal cancer patients are able to follow a low GL diet with an in-person dietary intervention. While the study participants provided positive feedback on the program, our recruitment data suggests that further program refinements may be needed to broadly attract patients to this type of intervention. The use of virtual dietitian visits may mitigate issues with travel and time constraints, and thus facilitate program scalability. 


\section{Acknowledgments}

This study was presented as a poster at the 2020 Virtual Annual meeting of the American Society of Clinical Oncology, abstract 4643.

Funding: This work was supported by the Clinical and Translational Science Collaborative of Cleveland, UL1TR002548 from the National Center for Advancing Translational Sciences (NCATS) component of the National Institutes of Health and NIH Roadmap for Medical Research. Its contents are solely the responsibility of the authors and do not necessarily represent the official views of the NIH. It was also supported by MetroHealth Cancer Center OROC \& Brett Altieri Memorial Funds, and philanthropic funding from Philip and Karen Swope.

\section{Footnote}

Reporting Checklist: The authors have completed the TREND reporting checklist. Available at: http://dx.doi. org/10.21037/jgo-20-330

Data Sharing Statement: Available at: http://dx.doi. org/10.21037/jgo-20-330

Peer Review File: Available at: http://dx.doi.org/10.21037/ jgo-20-330

Conflicts of Interest: All authors have completed the ICMJE uniform disclosure form (available at: http://dx.doi. org/10.21037/jgo-20-330). NJM reports a disclosure from Flatiron Health and Roche. The other authors have no conflicts of interest to declare.

Ethical Statement: The authors are accountable for all aspects of the work in ensuring that questions related to the accuracy or integrity of any part of the work are appropriately investigated and resolved. The study was approved by the Institutional Review Board at Seidman Cancer Center, University Hospitals Cleveland Medical Center, and utilized the Reliant Review at Metrohealth Medical Center (University Hospitals 01-14-07C, MetroHealth 15-00732). The study was conducted in accordance with the Declaration of Helsinki (as revised in 2013). Patients were approached at the time of their follow up visits or contacted by phone. Those identified through the tumor registry were contacted by phone and if interested were mailed a consent form and a 3-day dietary food record.
They were then contacted to review the consent form and if interested, consented by phone and were contacted by a member of the bionutrition team, who provided detailed instructions on how to complete a 3-day dietary intake log and return it for screening and evaluation. If eligible, an appointment with the dietitian was made either alone or in a small group, and in-person written informed consent was obtained at that time for each participant.

Open Access Statement: This is an Open Access article distributed in accordance with the Creative Commons Attribution-NonCommercial-NoDerivs 4.0 International License (CC BY-NC-ND 4.0), which permits the noncommercial replication and distribution of the article with the strict proviso that no changes or edits are made and the original work is properly cited (including links to both the formal publication through the relevant DOI and the license). See: https://creativecommons.org/licenses/by-nc-nd/4.0/.

\section{References}

1. Global Cancer Observatory [Internet]. [cited $2020 \mathrm{Feb}$ 27]. Available online: https://gco.iarc.fr/

2. Edge SB, Compton CC. The American Joint Committee on Cancer: the 7th edition of the AJCC cancer staging manual and the future of TNM. Ann Surg Oncol 2010;17:1471-4.

3. Schmoll HJ, Tabernero J, Maroun J, et al. Capecitabine plus oxaliplatin compared with fluorouracil/folinic acid as adjuvant therapy for stage III colon cancer: Final Results of the NO16968 randomized controlled phase III trial. J Clin Oncol 2015;33:3733-40.

4. André T, De Gramont A, Vernerey D, et al. Adjuvant fluorouracil, leucovorin, and oxaliplatin in stage II to III colon cancer: Updated 10-year survival and outcomes according to BRAF mutation and mismatch repair status of the MOSAIC study. J Clin Oncol 2015;33:4176-87.

5. André T, Boni C, Navarro M, et al. Improved overall survival with oxaliplatin, fluorouracil, and leucovorin as adjuvant treatment in stage II or III colon cancer in the MOSAIC trial. J Clin Oncol 2009;27:3109-16.

6. Meyerhardt JA. Beyond standard adjuvant therapy for colon cancer: role of nonstandard interventions. Semin Oncol 2011;38:533-41

7. Alberts SR, Sargent DJ, Nair S, et al. Effect of oxaliplatin, fluorouracil, and leucovorin with or without cetuximab on survival among patients with resected stage III colon cancer: a randomized trial. JAMA 2012;307:1383-93. 
8. Allegra CJ, Yothers G, O'Connell MJ, et al. Phase III trial assessing bevacizumab in stages II and III carcinoma of the colon: results of NSABP protocol C-08. J Clin Oncol 2011;29:11-6.

9. de Gramont A, Van Cutsem E, Schmoll HJ, et al. Bevacizumab plus oxaliplatin-based chemotherapy as adjuvant treatment for colon cancer (AVANT): a phase 3 randomised controlled trial. Lancet Oncol 2012;13:1225-33.

10. Meyerhardt JA, Sato K, Niedzwiecki D, et al. Dietary glycemic load and cancer recurrence and survival in patients with stage III colon cancer: findings from CALGB 89803. J Natl Cancer Inst 2012;104:1702-11.

11. Meyerhardt JA, Niedzwiecki D, Hollis D, et al. Association of dietary patterns with cancer recurrence and survival in patients with stage III colon cancer. JAMA 2007;298:754-64.

12. Choi Y, Giovannucci E, Lee JE. Glycaemic index and glycaemic load in relation to risk of diabetes-related cancers: a meta-analysis. Br J Nutr 2012;108:1934-47

13. Dray X, Boutron-Ruault MC, Bertrais S, et al. Influence of dietary factors on colorectal cancer survival. Gut 2003;52:868-73.

14. Kaaks R, Toniolo P, Akhmedkhanov A, et al. Serum C-peptide, insulin-like growth factor (IGF)-I, IGFbinding proteins, and colorectal cancer risk in women. $\mathrm{J}$ Natl Cancer Inst 2000;92:1592-600

15. Chi F, Wu R, Zeng YC, et al. Circulation insulin-like growth factor peptides and colorectal cancer risk: an updated systematic review and meta-analysis. Mol Biol Rep 2013;40:3583-90.

16. Giovannucci E. Insulin, insulin-like growth factors and colon cancer: a review of the evidence. J Nutr 2001;131:3109S-20S.

17. Vigneri PG, Tirrò E, Pennisi MS, et al. The insulin/IGF system in colorectal cancer development and resistance to therapy. Front Oncol 2015;5:230.

18. Gallagher EJ, LeRoith D. The proliferating role of insulin and insulin-like growth factors in cancer. Trends Endocrinol Metab 2010;21:610-8.

19. Sridhar SS, Goodwin PJ. Insulin-insulin-like growth factor axis and colon cancer. J Clin Oncol 2009;27:165-7.

20. Vander Heiden MG, Cantley LC, Thompson CB. Understanding the Warburg effect: the metabolic requirements of cell proliferation. Science 2009;324:1029-33.

21. Berger NA. Obesity and cancer pathogenesis. Ann N Y Acad Sci 2014;1311:57-76.

22. Pakiet A, Kobiela J, Stepnowski P, et al. Changes in lipids composition and metabolism in colorectal cancer: A review. Lipids Health Dis 2019;18:29.

23. Schoen RE, Tangen CM, Kuller LH, et al. Increased blood glucose and insulin, body size, and incident colorectal cancer. J Natl Cancer Inst 1999;91:1147-54.

24. Iyengar NM, Gucalp A, Dannenberg AJ, et al. Obesity and Cancer Mechanisms: Tumor Microenvironment and Inflammation. J Clin Oncol 2016;34:4270-6.

25. Iyengar NM, Brown KA, Zhou XK, et al. Metabolic Obesity, Adipose Inflammation and Elevated Breast Aromatase in Women with Normal Body Mass Index. Cancer Prev Res (Phila) 2017;10:235-43.

26. Sinicrope FA, Foster NR, Yothers G, et al. Body mass index at diagnosis and survival among colon cancer patients enrolled in clinical trials of adjuvant chemotherapy. Cancer 2013;119:1528-36.

27. Renfro LA, Loupakis F, Adams RA, et al. Body Mass Index Is Prognostic in Metastatic Colorectal Cancer: Pooled Analysis of Patients From First-Line Clinical Trials in the ARCAD Database. J Clin Oncol 2016;34:144-50.

28. Brown JC, Meyerhardt JA. Obesity and Energy Balance in GI Cancer. J Clin Oncol 2016;34:4217-24.

29. Bowen DJ, Beresford SA. Dietary interventions to prevent disease. Annu Rev Public Health 2002;23:255-86.

30. Ammerman AS, Lindquist CH, Lohr KN, et al. The efficacy of behavioral interventions to modify dietary fat and fruit and vegetable intake: a review of the evidence. Prev Med 2002;35:25-41.

31. Chlebowski RT, Reeves MM. Weight Loss Randomized Intervention Trials in Female Cancer Survivors. J Clin Oncol 2016;34:4238-48.

32. Ma Y, Olendzki BC, Pagoto SL, et al. Number of 24-Hour Diet Recalls Needed to Estimate Energy Intake. Ann Epidemiol 2009;19:553-9.

33. Schakel S, Schauer R, Himes J, et al. Development of a glycemic index database for dietary assessment. J Food Compos Anal 2008;21:S50-5.

34. Schakel SF. Maintaining a Nutrient Database in a Changing Marketplace: Keeping Pace with Changing Food Products-A Research Perspective. J Food Compost Anal 2001;14:315-22.

35. Schakel SF, Buzzard IM, Gebhardt SE. Procedures for estimating nutrient values for food composition databases. J Food Compos Anal 1997;10:102-14.

36. Johnson RK, Driscoll P, Goran MI. Comparison of multiple-pass 24-hour recall estimates of energy intake with total energy expenditure determined by the doubly labeled water method in young children. J Am Diet Assoc 
1996;96:1140-4.

37. Berkow SE, Barnard N, Eckart J, et al. Four therapeutic diets: adherence and acceptability. Can J Diet Pract Res 2010;71:199-204.

38. Katcher HI, Ferdowsian HR, Hoover VJ, et al. A worksite vegan nutrition program is well-accepted and improves health-related quality of life and work productivity. Ann Nutr Metab 2010;56:245-52.

39. Barnard N, Scherwitz L, Ornish D. Adherence and acceptability of a low-fat, vegetarian diet among patients with cardiac disease. J Cardiopulm Rehabil 1992;12:423-31.

40. Barnard ND, Gloede L, Cohen J, et al. A low-fat vegan diet elicits greater macronutrient changes, but is comparable in adherence and acceptability, compared with a more conventional diabetes diet among individuals with type 2 diabetes. J Am Diet Assoc 2009;109:263-72.

Cite this article as: Treasure M, Thomas A, Ganocy S, Hong A, Krishnamurthi SS, Bajor DL, Berger NA, Meropol NJ. A pilot study of a low glycemic load diet in patients with stage I-III colorectal cancer. J Gastrointest Oncol 2021;12(3):910-920. doi: 10.21037/jgo-20-330
41. Wulaningsih W, Garmo H, Holmberg L, et al. Serum Lipids and the Risk of Gastrointestinal Malignancies in the Swedish AMORIS Study. J Cancer Epidemiol 2012;2012:792034.

42. Ma J, Pollak MN, Giovannucci E, et al. Prospective study of colorectal cancer risk in men and plasma levels of insulin-like growth factor (IGF)-I and IGF-binding protein-3. J Natl Cancer Inst 1999;91:620-5.

43. Bashshur R, Doarn CR, Frenk JM, et al. Telemedicine and the COVID-19 Pandemic, Lessons for the Future. Telemed J E Health 2020;26:571-3.

44. Using Telehealth to Expand Access to Essential Health Services during the COVID-19 Pandemic I CDC. Cited 2020 Aug 10. Available online: http://www.cdc.gov/ coronavirus/2019-ncov/hcp/telehealth.html 